\title{
Desarrollo de la Movilidad Urbana de Medellín, Colombia, un Ejemplo para Costa Rica
}

Development of the Urban Mobility of Medellín, Colombia, an Example for Costa Rica Marcos Antonio Montenegro-Méndez ${ }^{1}$

Montenegro-Méndez, M.A. Desarrollo de la Movilidad Urbana de Medellín, Colombia, un Ejemplo para Costa Rica. Tecnología en Marcha. Edición especial. Movilidad Estudiantil 6, 2019. Pág 69-82

doi) https://doi.org/10.18845/tm.v32i8.4565 


\title{
Palabras clave
}

Movilidad Urbana; Transporte; Desarrollo; Medellín; Colombia; Costa Rica.

\section{Resumen}

La movilidad urbana es un factor inherente de la vida cotidiana, su desarrollo permite generar mejores condiciones de vida y su olvido conlleva a un inminente colapso de la ciudad. Hoy, San José en Costa Rica, se encuentra en una etapa de urgente intervención, las soluciones de movilidad propuestas; metro, tranvía y tren rápido compiten entre sí y reflejan el estado del desarrollo poco planificado de la ciudad. Se toma el modelo de Medellín en Colombia, que actualmente cuenta con un sistema integrado de transporte y movilidad urbana por medio de metro, tranvía, metrocables (teleféricos), metroplús (buses articulados), servicios alimentadores e integrados, para servir de ejemplo a San José. Su desarrollo no solo destaca los modos de transportarse, sino también las adecuaciones en espacio y accesibilidad que se traduce a un mejor aprovechamiento y mayor apropiación, mejorando la calidad de vida de quienes se ven beneficiados por estos servicios. Por medio de esta investigación de campo realizada con entrevistas y consultas bibliográficas, se busca orientar una solución a la problemática que presenta actualmente la ciudad de San José, tomando como ejemplo el interesante caso de desarrollo en movilidad urbana que representa Medellín. Se concluye con este texto que las soluciones para la situación de San José se deben generar a partir del ideal de integración, así como que Medellín es un ejemplo adecuado y potencial fuente de asesoramiento para los esfuerzos de Costa Rica.

\section{Keywords}

Urban Mobility; Transport; Development; Medellín; Colombia; Costa Rica.

\begin{abstract}
Urban mobility is an inherent factor in daily life, its development allows better life conditions and its forget brings an imminent collapse of the city. Today, San José in Costa Rica, is in a time of urgent intervention, the proposed mobility solutions; metro, tram and rapid train are competing between it selves and shows the state of development little planned of the city. Medellín in Colombia is taken as a model that currently has an integrated system of transportation and urban mobility by metro, tram, metrocables (cable railway), metroplús (articulated bus), feeding and integrating systems, to serve as an example to San José. Its development not only distinguish the transportation ways, but also the space and accessibility adequacy that is translated to a better use and appropriation, getting a better life quality for every person who is involve in those services. Through this field investigation done with interviews and bibliography research, its pursuit to orientate a solution to San José's problematic situation, using such as example the interesting case of development in urban mobility that represents Medellín. Its concluded with this text that solutions to San José's situation must be generated starting the ideal of integration, also Medellín is an appropriate example and potential source of advice for Costa Rica's efforts.
\end{abstract}




\section{Introducción}

La rama particular de la ingeniería de transportes es definida por el Colegio Federado de Ingenieros y de Arquitectos de Costa Rica (CFIA) entre los perfiles profesionales como un "campo profesional en el cual se construye conocimiento a partir de las disciplinas matemáticas, de las ciencias naturales y de las ciencias sociales, (...) para su aplicación ética en el desarrollo de métodos, de utilización de materiales, esfuerzos y sinergia, dentro del marco del desarrollo sostenible, para satisfacción de las necesidades de movilización de la sociedad" [1]. En el marco del proyecto que a continuación se presenta, la definición anterior es de importancia ya que se refiere a la ingeniería como un pilar para el desarrollo de las ciudades y la intervención del entorno.

Sin embargo, tal intervención no ha sido profundamente interiorizada en algunos casos, ya que la ingeniería civil es en aplicación, transformadora del entorno en todas sus escalas y en todos sus ámbitos. Es particularmente la ingeniería de transportes, la rama de esta disciplina que podría verse más adherida a la interpretación anterior, debido a que su función es, en síntesis, comunicar puntos de interés para posibilitar acciones particulares entre las comunidades. En una Costa Rica que al año 2017 es agobiada por la situación caótica del tráfico, escasa inversión en movilidad urbana y pérdida de vista del espacio público no es superfluo extender los horizontes para referir al avance que en otras naciones y ciudades se realiza en búsqueda de dar solución a problemáticas similares dentro del entorno propio de cada región.

En el contexto planteado con las líneas anteriores, este documento tiene el objetivo de orientar una solución a la problemática que presenta actualmente la ciudad de San José, en Costa Rica, tomando como ejemplo el interesante caso de desarrollo en movilidad urbana que representa Medellín, en Colombia.

\section{Métodos y Fuentes de Información}

Para el alcance del objetivo planteado en este proyecto, fue requerido el acceso de información mediante diferentes métodos y técnicas, consultado distintas fuentes y sujetos. Entre los métodos aplicados a la investigación de campo se encuentra la entrevista, revisión documental y consulta bibliográfica, la observación y visitas técnicas.

Por su parte, en las fuentes de información se destacan la Empresa de Transporte Masivo del Valle de Aburrá Ltda. (Metro de Medellín Ltda.), la biblioteca del Metro y empresas del sector, así como los sujetos de información se centran en profesionales del medio, relacionados a las intervenciones de planeación, dirección y coordinación de ingeniería en transportes del Metro de Medellín.

\section{Resultados y Discusión}

A continuación, se muestran los resultados obtenidos en respuesta al objetivo planteado para esta investigación, ahondando de inmediato en su discusión, interpretación y análisis. Los resultados se dividen en tres grupos principales, a saber; contexto general de comparación, la problemática de San José, y el caso de Medellín.

\section{Contexto General de Comparación}

Con la intención de exponer a Medellín y su desarrollo durante las últimas décadas como un modelo para San José, esta sección se aplica a detallar algunos datos macro de ambas ciudades y países, de tal forma que se obtiene un escenario donde se permite la comparación y homologación de las vivencias, experiencias y situación entre las dos ciudades en aspectos que resultan de interés para el enfoque de la investigación desarrollada. 


\section{San José, Costa Rica}

Costa Rica es un país de 51.100 kilómetros cuadrados de territorio y una población proyectada que al año 2017 supera los 4.900.000 habitantes de acuerdo con datos del Instituto Nacional de Estadística y Censo [2]. La provincia de San José ostenta la capital del país desde el año 1823, cuenta con una extensión de 4.959,63 kilómetros cuadrados y concentra casi una tercera parte de la población del país, su cantón central que lleva el mismo nombre se extiende en 44,62 kilómetros cuadrados, pero destaca ya que conforma junto a otros cantones de la misma provincia y de las provincias de Cartago, Alajuela y Heredia el Gran Área Metropolitana (GAM), llegando a una extensión de 1.779 kilómetros cuadrados y una concentración aproximada del $50 \%$ de la población total en el país.

\section{Medellín, Colombia}

El país suramericano de Colombia tiene casi 20 veces la extensión territorial de Costa Rica (1.141.748 kilómetros cuadrados), y ubica dentro del departamento de Antioquia al Valle de Aburrá; conformado entre otros por los municipios de Bello, Medellín, Envigado, Itagüí, Sabaneta y La Estrella. El Valle de Aburrá tiene un territorio de 1.152 kilómetros cuadrados, y su población proyectada para el año 2015 rondaba los 3.777.000 habitantes, de acuerdo con datos de la Cámara de Comercio de Medellín para Antioquia [3]. Por su parte, Medellín es el municipio que alberga la capital del departamento de Antioquia, tiene una extensión de 383 kilómetros cuadrados y una población proyectada de 2.434.647 habitantes para el año 2015 según datos de la CCMA.

\section{La Problemática de San José, Costa Rica}

La capital de cada país es en común, el punto de mayor concentración de personas por las múltiples oportunidades, servicios y facilidades que ofrece; como empleos, salud, educación, transporte, recreación entre otros. San José retiene en transporte uno de los puntos medulares de sus problemáticas actuales como ciudad, entre las soluciones que se presentan para dicha situación se hayan diferentes propuestas como un sistema de metro, tranvía y tren rápido de pasajeros (TRP), y aunque estas alternativas se han presentado en un escenario fundamentalmente excluyente, es meritorio prestar atención a las soluciones que en otros países se han dado y donde se ve converger las ventajas de los distintos medios de transporte.

\section{El Caso de Medellín, Colombia}

A pesar de haber sufrido los embates del conflicto armado en Colombia, Medellín ha demostrado al mundo la capacidad de resiliencia de su gente al punto de ser una ciudad modelo y protagonista en desarrollo tanto de América Latina, como del mundo.

En conocimiento de la situación que vive actualmente Costa Rica en su ciudad capital, se descubre al fijar la mirada en esa región de Suramérica que hace más de dos décadas tomó el reto del desarrollo y sacándole ventaja, ahora representa un ejemplo bien ajustado para la medida de Costa Rica.

\section{El Transporte y la Movilidad Urbana en el Área Metropolitana}

Tanto los medellinenses como cualquier visitante; local o extranjero, puede favorecerse de las ventajas que brinda la ciudad en cuanto a transporte y movilidad urbana. Con varias décadas de desarrollo hacia un modelo de crecimiento estructurado para el Área Metropolitana, tanto en transporte como en su infraestructura, Medellín ofrece a los ciudadanos diversos medios de movilidad mediante un sistema integrado de transporte, donde convergen las acciones de 
servicios masivos, colectivos e individuales para dar mayor fluidez al trasladarse, contribuyendo al ambiente y generando mejoras en la calidad de vida de las personas.

A continuación, se evidencia la experiencia de Medellín mediante la creación, operación y gestión de sus principales actores en movilidad urbana: metro, tranvía, cables teleféricos y buses de tránsito rápido, aunados a soluciones complementarias que refuerzan las acciones ya tomadas y orientan el crecimiento de la ciudad, tal que estos sirvan como modelo y guía para los esfuerzos de Costa Rica en el tema de movilidad y transporte urbano.

Metro de Medellín (Línea A y B)

Desde el 30 de noviembre del año 1995 Medellín cuenta con un metro que recorre la ciudad en un eje principal Norte - Sur, escoltado en algunos tramos por el río Medellín y en otros, irrumpiendo en la ciudad con sus rieles en un viaducto elevado de doble vía. Bajo la administración de la Empresa de Transporte Masivo del Valle de Aburrá Limitada (Metro de Medellín Ltda.) que se creó en 1979 con la finalidad de planear, construir, administrar y operar el sistema de transporte público masivo, el metro es la columna vertebral de la movilidad urbana en esta ciudad.

Su trazado original se extendía a lo largo del eje natural de río Medellín en la Línea A, entre los municipios de Itagüí y Bello con las estaciones Poblado y Niquía respectivamente, y la línea B ingresaba al centro de la ciudad en un viaducto hasta el parque Berrío según el diseño preliminar de 1980 [4]. Finalmente, este trazado fue modificado para dar paso a la configuración actual de las dos líneas del metro tal como se muestra en la figura 1, cruzando parte del Valle de Aburrá en un corredor a nivel de calle en sus extremos y en viaducto elevado sobre el centro de la ciudad de Medellín.

En la línea A el metro cuenta con 21 estaciones, mientras la línea B presenta 7 estaciones más (incluida la conexión de transferencia en San Antonio), y sobre ambas transita una flotilla de 77 trenes, considerando los recientes equipos adquiridos en España con los cuales se alcanzaría una flota de 80 trenes en total. Se estima que por algunas de sus estaciones pueden reportarse aproximadamente 42.000 usuarios a diario, lo que evidencia un impacto significativo en la necesidad real de trasladarse de las personas con el fin de realizar sus actividades cotidianas, pero que, a la vez destaca en el aporte agregado en temas de eficiencia y sostenibilidad, ya que el sistema metro se alimenta de energías limpias, reduce la contaminación sónica y del aire, y brinda un servicio confiable, seguro y constante para atender a la población.

Aunque los trenes del metro son el eje central del sistema de transporte masivo de personas en Medellín, estos se acompañan de otros medios del Sistema Metro que se detallan adelante, y que en conjunto trasladan un promedio de 811.000 personas por día, reportando para el año 2016 que se logró brindar servicio a cerca de 270 millones de usuarios, con la cifra pico de más de un millón de pasajeros en un día típico laboral [5].

Entre las facilidades que se le pueden destacar a este servicio, es el pago electrónico para ingresar a las estaciones, la principal herramienta para esta transacción es la "tarjeta Cívica" con la cual se permite el acceso a todas las estaciones del Sistema Metro. De acuerdo con el Metro de Medellín [6], actualmente se cuenta con más de mil puntos para la recarga de la tarjeta Cívica adicionales a las estaciones y paradas, debido a que dicha tarjeta funciona por medio de almacenamiento de dinero digital, que se debita cada vez que se accede a algún modo de transporte del Sistema Metro, la recarga externa permite desahogar la demanda en las taquillas de las estaciones, agilizando el acceso al servicio y brindando mejores comodidades a los usuarios por aprovechamiento efectivo del tiempo. Por otra parte, también se puede acceder al sistema mediante el uso de tiquetes y tarjetas desechables, que permiten el uso del metro para viajes específicos, o bien para poblaciones temporales como es el caso de turistas. 


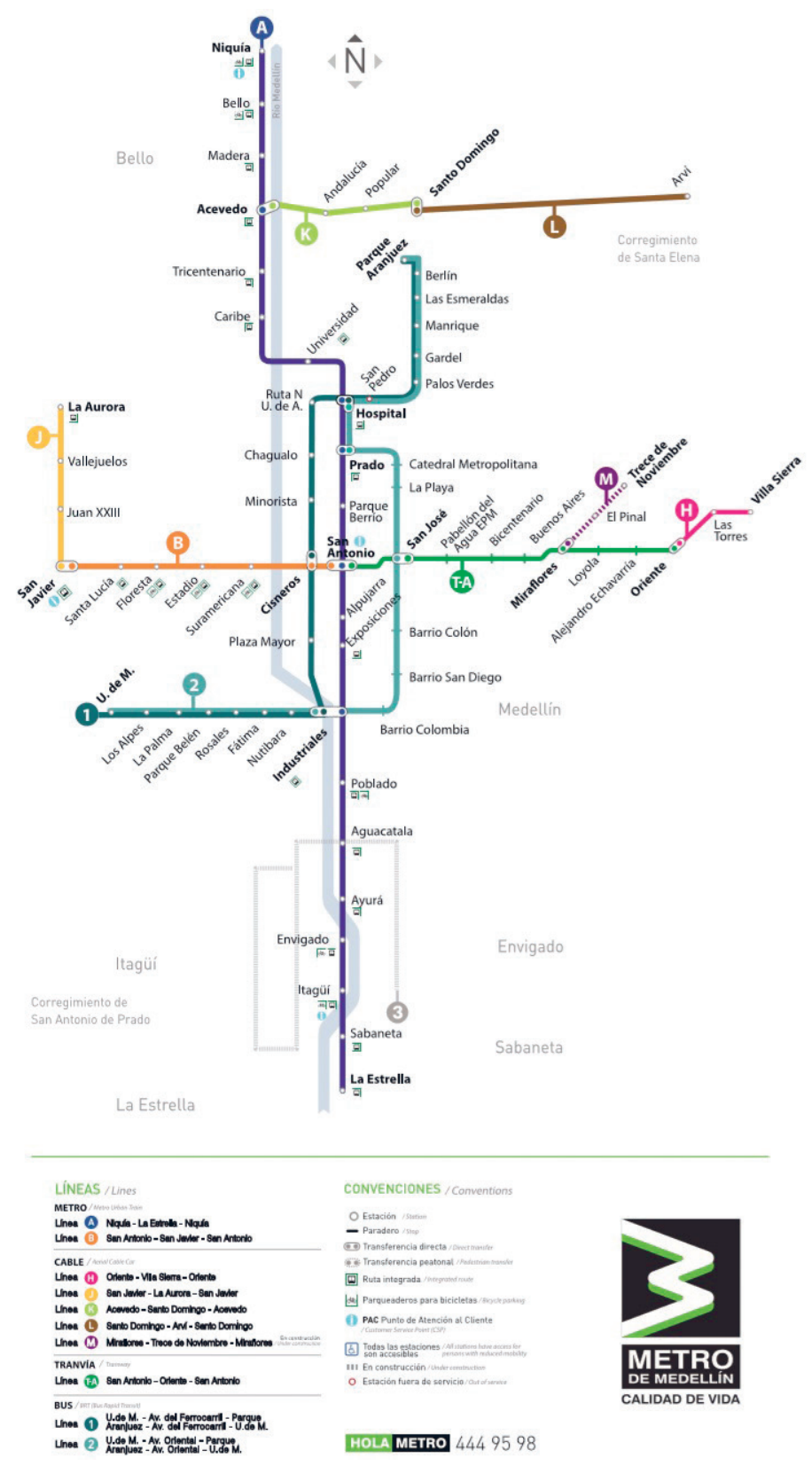

Figura 1. Mapa esquemático del Metro de Medellín 2017. Fuente: Metro de Medellín Ltda.

Así mismo, es meritorio resaltar la estructura de las estaciones, apoyados en la figura 1, desde la estación La Estrella al sur hasta la estación Industriales los rieles se encuentran a nivel de vía, viajando paralelo al cauce del río Medellín por uno u otro costado, debido que en dicho tramo los rieles cruzan el río en dos oportunidades. Y por su estructura algunas de las estaciones se encuentran andenes laterales a los rieles, o un único andén central. Desde la estación Exposiciones hasta Universidad, el metro viaja en viaducto elevado con doble andén paralelo a los rieles es todas las estaciones, y bajo esta condición de viaducto se configura también la línea B. Finalmente, desde la estación Caribe hasta Niquía, el metro regresa a nivel de vía replicando las características del tramo sur en cuanto a la configuración de sus estaciones.

Es importante señalar también que, a pesar del mantenimiento que reciben las máquinas como las vías y estaciones, actualmente se trabaja en un plan operativo para brindar un mejor servicio 
a los usuarios que involucra el aumento en la frecuencia del servicio y la ampliación particular de la estación Poblado, debido a que es una de las estaciones más congestionadas en horas pico. Por otra parte, resaltar la presencia de la sede administrativa del Metro de Medellín (ver figura 2) adyacente a la estación Bello, donde además se ubica el patio de mantenimiento de los trenes.

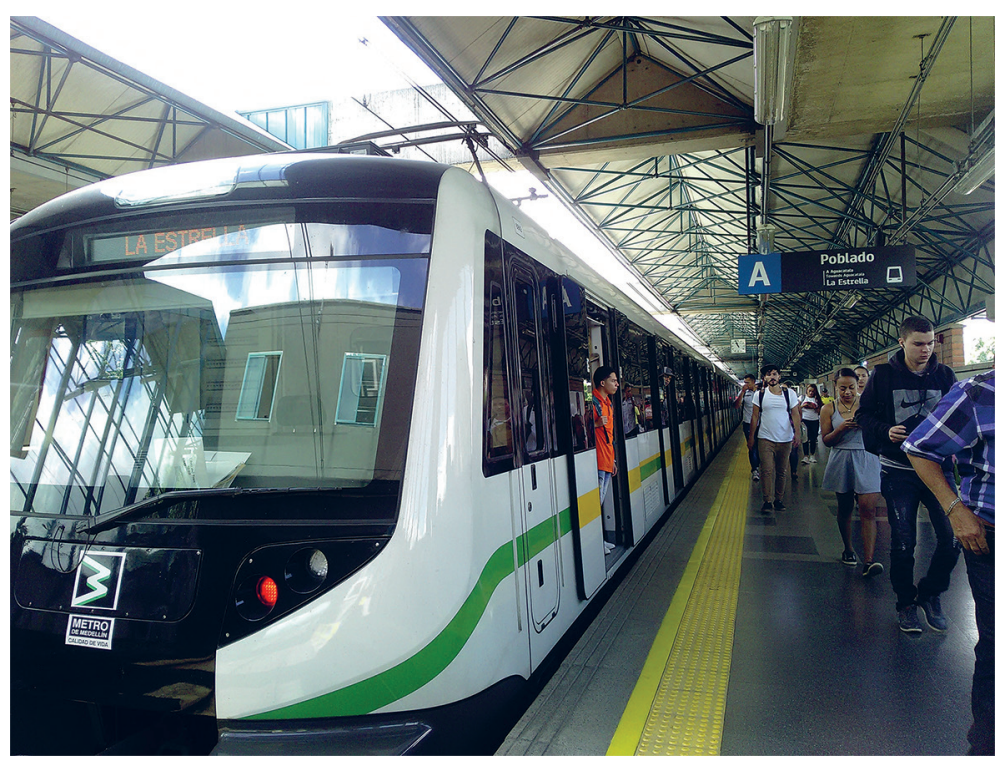

Figura 2. Estación Poblado del Metro de Medellín.

\section{Tranvía de Ayacucho (TA)}

El libro "Entre rieles y cables" [7] recuerda el inicio de las obras constructivas para uno de los tranvías más modernos en Latinoamérica el 4 de marzo del 2013, proceso que se extendió por más de dos años hasta dar el 15 de octubre del 2015 con la primera interacción del tranvía y los ciudadanos y la apertura del servicio comercial al finalizar noviembre del mismo año. Este servicio de transporte público permite trasladar pasajeros desde la transferencia o conexión con la estación San Antonio del metro, en el centro de la ciudad, hasta la estación Oriente ubicada a 4,3 kilómetros de distancia.

Su servicio presenta diversas particularidades, entre ellas se encuentra que el corredor del tranvía es exclusivo para modos de transporte sostenibles, ya que se comparte con ciclovías e interactúa con áreas de esparcimiento completamente renovadas. Se suman también, las características de los coches; 5 vagones de 7 metros de longitud cada uno con 12 neumáticos de caucho a cada lado y un riel central como guía. Además, el tranvía se alimenta de energía eléctrica por medio de catenaria de forma permanente que habilita al coche para trasladarse a una velocidad promedio de 30 kilómetros por hora, lo que aporta a los esfuerzos por el uso de energías amigables con el ambiente. Aunado a lo anterior, es remarcable que la capacidad del tranvía permite movilizar hasta 300 persona en un viaje, con frecuencias aproximadas de 4 minutos en las horas más concurridas del día, al tiempo que en las estaciones de este servicio, el usuario presenta completa autonomía de ingreso empleando la tarjeta Cívica del metro, y la estructura de las estaciones permiten el acceso a la estación y a los vagones por medio de puertas corredizas a media altura, ubicadas en un doble andén que rodea los rieles del tranvía.

Como un servicio integrado a la línea $A$ del metro, el tranvía recupera también la "Cultura Metro", una acción e iniciativa social que busca generar en los usuarios la apropiación de 
todos los modos de transporte del Sistema Metro, y que, según el ingeniero Germán Madrid [8], empezó cerca de dos años antes de la apertura del metro al servicio comercial. Mediante esta iniciativa se logra en los usuarios de los modos de transporte se conviertan en inspectores de un correcto funcionamiento y comportamiento en las estaciones, paradas, vagones y cabinas, ayudando a conservar en mejores condiciones todas las instalaciones del sistema y mantener una interacción entre usuarios más saludable y de mayor compañerismo.

En el año 2016, se logró habilitar la interconexión del tranvía con uno de los dos metrocables que se tiene previsto, específicamente con la Línea H ubicado en la estación Oriente, con estos avances se posibilita la movilidad de cientos de pasajeros en tres modos del Sistema Metro (figura 3), facilitando su traslado por medio en transportes sostenibles, con eficiencia en los tiempos y mejor calidad del servicio, en contraste con otros modos motorizados.

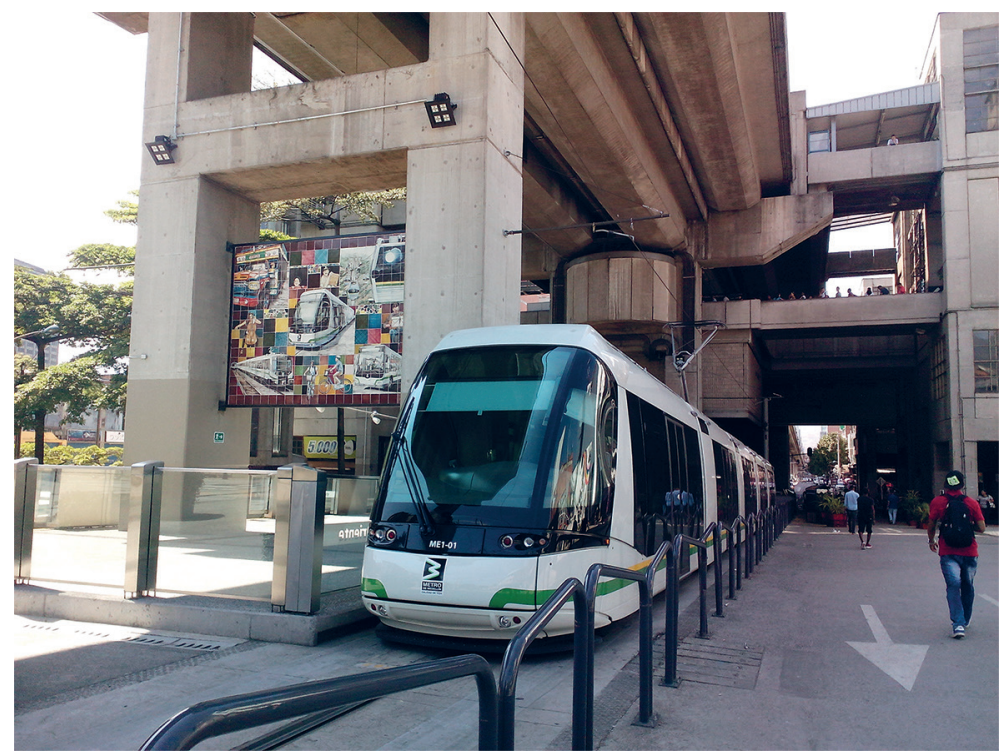

Figura 3. Conexión al tranvía en la estación San Antonio del Metro.

\section{Metrocables (Líneas $H, J, K, L$ y $M$ )}

Los metrocables son un sistema de transporte de personas tipo periférico, que opera desde el año 2004 cuando se habilitó la primera línea de cables, esta corresponde a la Línea K (figura 4) y sale de la transferencia en la estación Acevedo del Metro hasta la estación Santo Domingo, a unos dos kilómetros de distancia y aproximadamente 400 metros de elevación, para permitir mejor movilidad a los ciudadanos del sector más alejado al nororiente de Medellín. Luego, se crearían las Líneas J y L, en 2008 y 2010 respectivamente [9], donde la primera conduce por aproximadamente tres kilómetros a los pasajeros hasta la estación La Aurora, iniciando en la transferencia de la estación San Javier en la Línea B del Metro, y con la particularidad de ser un cable que no asciende de forma sostenida, sino que escala el sector de El Morro para luego descender y ascender de nuevo en el tramo final de la línea. Por su parte, la Línea $L$ corresponde a un cable exclusivamente turístico, debido a que su destino es el Parque Arví, ubicado en la vereda El Tambo al oriente de Medellín. Este metrocable tiene su transferencia al Metro en la estación Santo Domingo de la Línea K, y presenta la característica de ser el más extenso de los cuatro existentes actualmente, con 4,6 kilómetros de longitud.

Más recientemente, y como se mencionó en el apartado anterior, en el 2016 se inauguró el servicio comercial del metrocable Línea $\mathrm{H}$, con salida desde la conexión en la estación Oriente 
del Tranvía de Ayacucho (TA), y que traslada por 1,4 kilómetros a los pasajeros hasta la estación Villa Sierra, en el sector oriental del municipio de Medellín, con la particularidad de que su línea presenta un giro de 50 grados en la estación Las Torres. Aunado a estos cuatro cables, se encuentra el metrocable Línea M, que tiene conexión con la Línea TA en la estación Miraflores y asciende hasta la estación Trece de Noviembre, cerca de las faldas del cerro Pan de Azúcar, con un trayecto que cubre poco más de 1,0 kilómetro [7].

Es importante destacar que Medellín es la primera ciudad en el mundo en implementar el servicio de tipo teleférico como modo de transporte masivo de personas, y al estar integrado a los otros modos del Sistema Metro, se logra acercar a las poblaciones más alejadas de Medellín, ahorrando tiempo y dinero, a la vez que dinamiza la integración social en el Valle de Aburrá. Además, su puesta en marcha ha requerido del acercamiento y sensibilización de la población para un correcto uso de las cabinas, ya que el sistema opera de forma autónoma lo que quiere decir que el personal del metro se ubica únicamente en las estaciones. Por otra parte, los metrocables reciben mantenimiento de forma anual, para inspección y mejora de todos los sistemas involucrados; seguridad, control, socorro y motriz, revisando también los cables, la tensión de operación y las condiciones en prueba de frenado y potencia de acuerdo con la normativa internacional correspondiente y las normas ISO 9001 e ISO 14001 en las cuales se encuentra certificada la empresa Metro de Medellín Ltda. [10].

En la sección del libro "Entre rieles y cables" a cargo de Ignacio Piedrahita [7], Todo un reto de ingeniería, se recuerda también la particularidad de los metrocables con respecto a las pilonas metálicas que sirven de sujeción a los cables en su extremo superior, con diámetros de hasta dos metros y medio, las pilonas se colocan con ciertos grados de inclinación buscando la perpendicularidad entre la pendiente del terreno y su fuste, y así dar la capacidad de soportar las cargas propias de las cabinas y su uso.

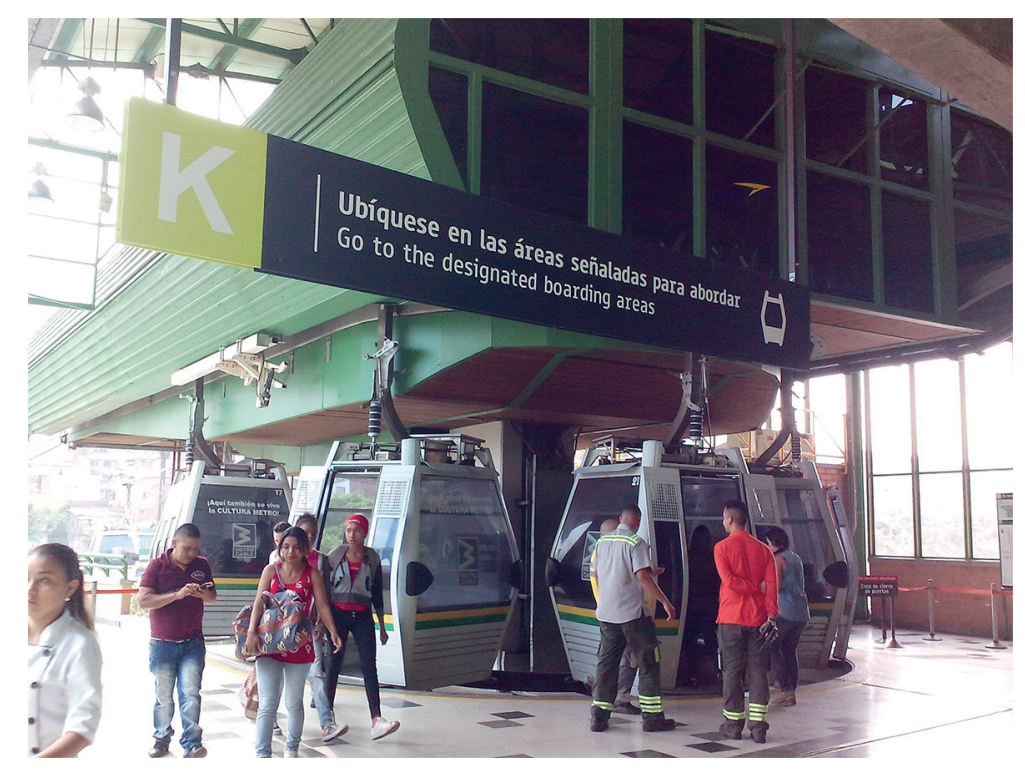

Figura 4. Metrocable Línea K, estación de transferencia Acevedo del Metro.

\section{Metroplús (Línea 1 y 2)}

Es un sistema de transporte masivo de mediana capacidad en el área metropolitana, creado al amparo de la Política Nacional de Transporte Urbano. Forma parte del Sistema Integrado de Transporte del Valle de Aburrá (SITVA), su administración y gestión corresponde a la entidad 
Metroplús S.A., aunque converge física, tarifaria y operacionalmente con distintos modos de transporte como el Metro en algunas de sus estaciones. El sistema de metroplús (figura 5) consta de buses articulados BRT (bus rapid transit) que brindan el servicio, y tiene semejanza con el sistema de transporte del Transmilenio en Bogotá, ente que acompañó el desarrollo del Metroplús para el Valle de Aburrá [11].

El servicio actual de metroplús cuenta con dos líneas en funcionamiento; la Línea 1 inicia su operación en el año 2011 y la línea 2 se inaugura en el año 2013. La Línea 1 recorre desde la Universidad de Medellín hasta el parque de Aranjuez por un carril exclusivo de 13,6 kilómetros de extensión, con 21 estaciones en su recorrido. Por su parte, la Línea 2 posee los mismos destinos terminales, pero se traslada por la ciudad sobre la Avenida Oriental en carriles compartidos con preferencia para servicios de autobús extendiéndose en 5,5 kilómetros, con un total de16 estaciones.

Con este servicio, se busca promover e incentivar el transporte masivo en el Valle de Aburrá, y tiene inferencia en los municipios de Medellín, Envigado e Itagüí. En estos dos últimos municipios se estrenarán años dos líneas de metroplús, aportando eficiencia en el sistema de transporte y la movilidad de los ciudadanos, reduciendo los tiempos de espera, y aportando valor agregado a la calidad de vida de las personas. Así mismo, el Metroplús funge un papel importante en la planeación, ejecución y control de la infraestructura y operación del servicio de transporte integrado de mediana capacidad en el Valle de Aburrá, no solamente participa en las rutas y estaciones del sistema Metroplús, sino que además gestiona la transformación de los espacios donde se ejecutan sus obras, dentro del marco del compromiso social, económico, social, cultural y de accesibilidad [11].

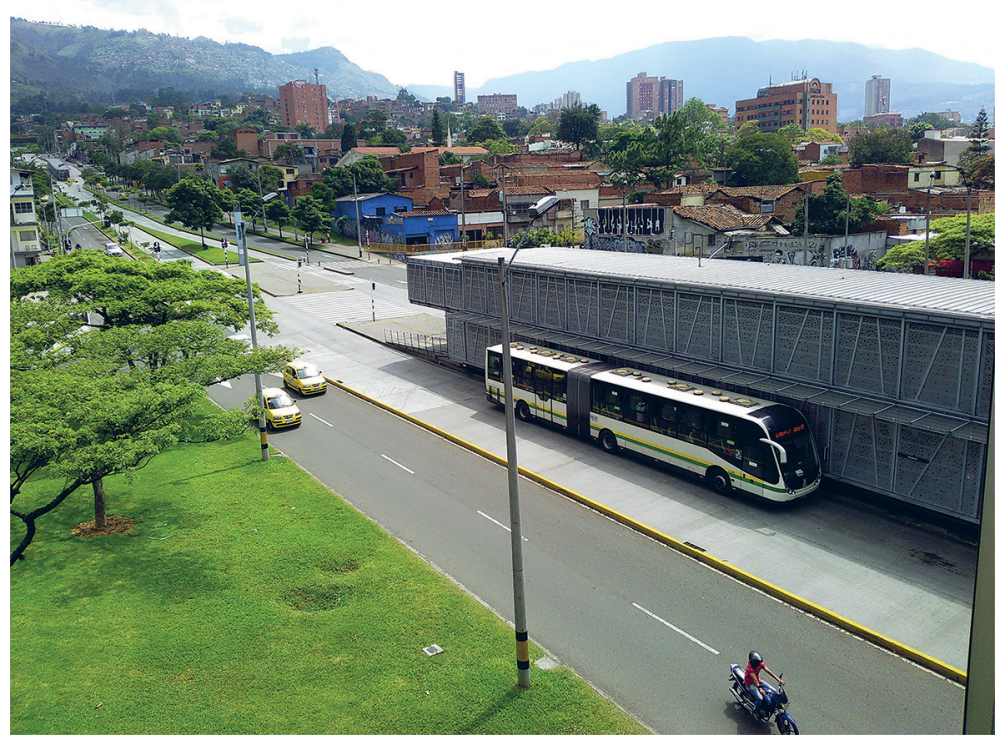

Figura 5. Metroplús en la transferencia de la estación Universidad del Metro.

\section{Alimentadores e Integrados (Transportes adjuntos)}

Se han desarrollado y adaptado como medios de accesibilidad a la red principal de movilidad en el Valle de Aburrá, su función es trasladar pasajeros por medio de microbuses desde puntos alejados hasta estaciones del Sistema Metro cercanas, para facilitar su desplazamiento a los diferentes destinos de la ciudad. 
Para el año 2014 se contaba con la presencia de 20 rutas alimentadoras y 130 rutas integradas como convenios comerciales, hoy en día ese número ha crecido con el desarrollo y evolución del Sistema Metro y del SITVA (ver figura 6) [12]. Tanto los servicios alimentadores como los integrados, permiten acortar distancias entre los usuarios y la red principal de movilidad urbana, en el caso particular de los microbuses alimentadores los caracteriza que su ingreso es efectuado únicamente con el uso de la tarjeta Cívica, de modo tal que se presenta la integración tarifaria con el fin de habilitar el acceso a dos o más modos de transporte con la cancelación de un único tiquete, o en su efecto, presentando tarifas reducidas. Los servicios integrados, pueden accederse mediante el uso de la tarjeta Cívica; cuando las rutas así están habilitadas, o mediante el pago de la tarifa en efectivo. Adicionalmente se cuenta con la opción de adquirir "tiquetes integrados", que permiten el acceso a las líneas del metro y a los servicios de microbuses integrados por una tarifa preferencial o reducida, dando también con un ahorro económico y mayor alcance en cuanto a los usuarios de la red de transporte. En ambos casos, el transitar de los vehículos de esta red se da en carriles compartidos con otros medios de transporte como automóviles, ingresando en los municipios a las distintas comunas que se circunscriben en su alcance.

Aunque en un inicio, la implementación de estas rutas de transporte reflejaba un ambiente confuso, la idea y misión de concatenar un servicio de calidad, eficiente e integrado ha traído mejores condiciones y alternativas para la movilidad de los ciudadanos, acompañado de un proceso de culturización en la población que toma gran importancia. La mayoría de estaciones del Sistema Metro tiene servicios de busetas alimentadoras y/o integradas, en ambos casos los vehículos que brindan el servicio por las distintas rutas se pueden ubicar en las cercanías de las estaciones.

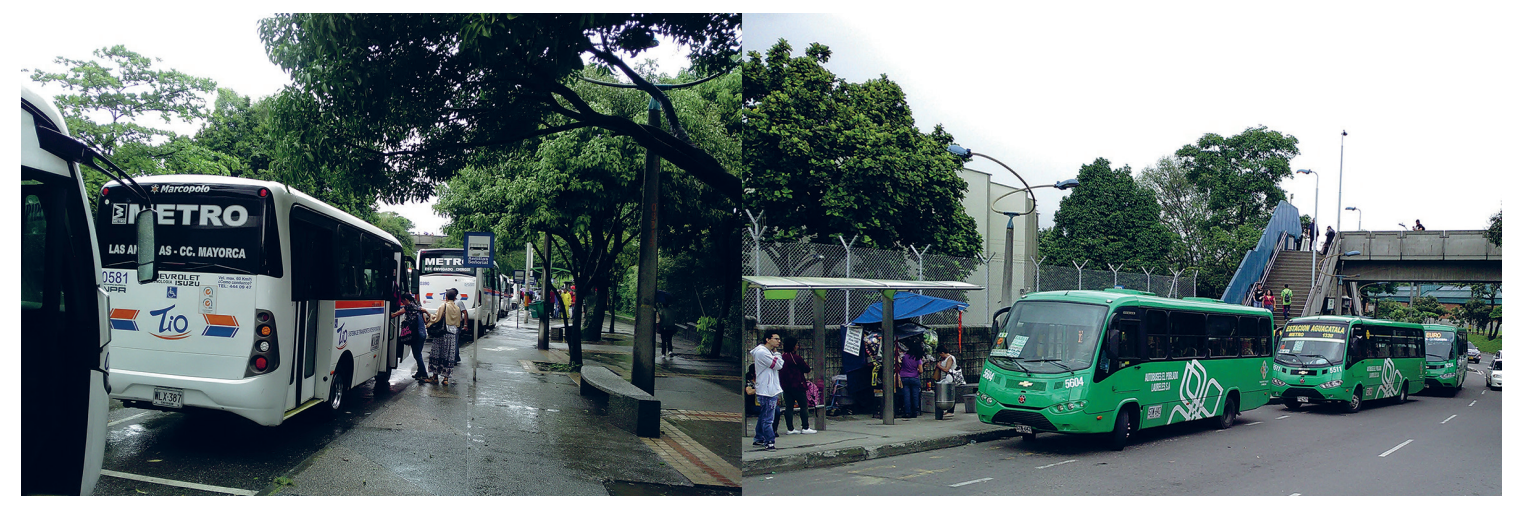

Figura 6. Sistema de Alimentadores del metro, estación Envigado (izq.)

e Integrados al metro, estación Aguacatala (der.).

\section{Otras facilidades de transporte}

Como medios complementarios a la movilidad y el transporte en el Valle de Aburrá, los ciudadanos cuentan con otras facilidades que permiten ordenar, vincular y atender de forma eficaz las necesidades de trasladarse. Entre estas facilidades, se puede destacar la presencia del sistema Encicla, las terminales de transporte, los servicios de buses "remanentes" metropolitanos, el transporte colectivo por medio de taxis.

Encicla es un sistema de transporte alternativo con bicicletas que funciona en algunas zonas del área metropolitana, con el mismo se apoya la iniciativa de la movilidad sostenible, la recreación y el deporte, buscando que los usuarios utilicen bicicletas como el modo de transportarse a sus destinos cotidianamente. En varias estaciones del metro, como por ejemplo la Estación 
Universidad, se encuentran puntos de acopio y atención a los usuarios, quienes por medio de la tarjeta Cívica pueden acceder al préstamo de una bicicleta para su uso, y desplazarse por las diferentes ciclovías que hay definidas en la ciudad. El préstamo se realiza por una duración de una hora con opción de renovar el préstamo en cualquier estación, con horarios que varían según el día entre las 5:30am y las 10:00pm [13]. Por otra parte, Medellín cuenta con dos grandes terminales de transporte, a saber; la Terminal Alberto Díaz Muñoz (Terminal del Sur, ver figura 7) ubicada en el sector de Poblado, cercana al aeropuerto Olaya Herrera y en operación desde abril del año 1993, y la Terminal Mariano Ospina Pérez (Terminal del Norte) que cuenta con transferencia directa a la Estación Caribe del Metro y brinda servicio desde febrero de 1984. Estas terminales de transporte brindan servicios a usuarios que buscan desplazarse a otras ciudades, pueblos o departamentos del país, conectando destinos a una, doce o más horas de distancia, y su ubicación estratégica a las afueras del centro de Medellín es un factor importante para no congestionar el tráfico a lo interno de la ciudad.

En cuanto a los servicios "remanentes" de buses en el área metropolitana, estos son las líneas de busetas y microbuses que operan en el Valle de Aburrá, pero que no están vinculadas con los servicios integrados por el Metro o el Metroplús, a la vez que no compiten por transportar usuarios a otras ciudades fuera del área metropolitana. Su servicio se enmarca en los modos de transporte colectivo de pasajeros (TCP) y sus rutas las comparten con otros vehículos como automóviles, motocicletas y los mismos integrados y alimentadores del Metro, permitiendo transportar entre 10 y 19 personas en cada viaje. Así mismo, el servicio colectivo de taxis se presta en toda el área metropolitana, y fácilmente se pueden ubicar zonas de taxis (acopios de taxis) en las afueras de las estaciones del Metro, cerca de centros comerciales y de puntos turísticos.

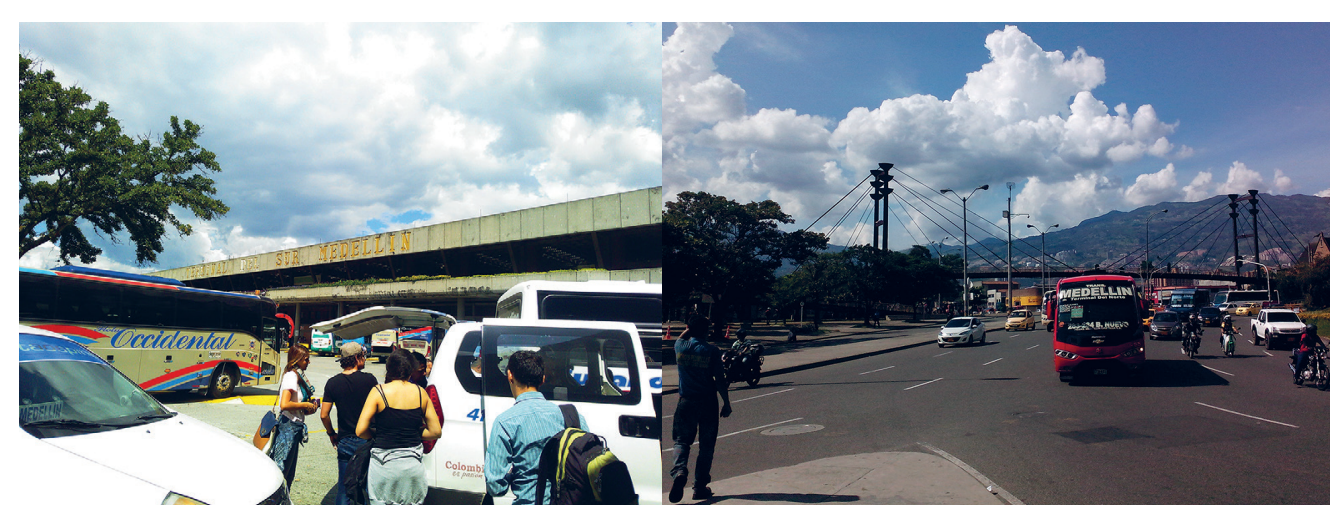

Figura 7. Terminal del Sur (izq.) y modos de transporte colectivo (der.).

\section{Conclusiones}

Habiendo realizado la presentación del caso modelo de desarrollo en cuanto a movilidad urbana de Medellín; considerando el Valle de Aburrá y el Área Metropolitana, se puede legar a las siguientes conclusiones como respuesta al objetivo planteado.

- La solución a la problemática que presenta San José en Costa Rica debe concebirse desde su inicio bajo el ideal de integración modal de transporte, como se ha ido consolidando en Medellín, Colombia. 
- Algunas soluciones que se exponen para la situación de San José ya se han aplicado en Medellín para combatir los males de congestión, movilidad y calidad de vida, así Medellín es un ejemplo de gran utilidad para proyectar la dirección de acciones y pautas a tomar en Costa Rica.

- La experiencia de Medellín como ciudad que cuenta con distintos modos integrados a una red de transporte masivo de personas, para facilitar la movilidad urbana y el desplazamiento dentro del Valle de Aburrá le retribuye importancia como potencial asesor de las medidas a tomar en Costa Rica.

\section{Recomendaciones}

El estudio llevado a cabo, permite realizar un levantamiento del desarrollo y gestión llevado a cabo en Medellín para lograr una movilidad urbana adecuada, al tiempo que introduce en breve el estado general del GAM en Costa Rica y presenta algunos rubros que permitan comparar ambos casos, pero las limitaciones que circundan esta investigación de campo se reflejan en una serie de recomendaciones para futuras investigaciones que tomen el ejemplo de Colombia y lo contrasten con la experiencia costarricense, mismas que se muestran a continuación.

- Se sugiere realizar el estudio de las ciudades colombianas de Bogotá, Cartagena y Pereira, que poseen un servicio de buses articulados (BRT) como soluciones de transporte de personas de mediana capacidad.

- Efectuar una revisión de los Planes de Ordenamiento Territorial (POT) en Medellín y los municipios interconectados por el Sistema Metro, así como los Planes de Movilidad (PM) propios de la Empresa de Transporte Masivo del Valle de Aburrá Ltda. como estructuras de gran provecho en la orientación del desarrollo en las ciudades.

- Estudiar las acciones en Medellín para lograr una ciudad sostenible a futuro; dentro de las cuales se destaca la mitigación en el uso de derivados del petróleo, y la apuesta por energías renovables.

- Realizar el levantamiento de detalle en cuanto a la intervención del espacio público en la ciudad de Medellín, con el fin de determinar su impacto real en la calidad de vida de la población, su desarrollo y los mecanismos de gestión que se apliquen.

- Ahondar en el estudio puntual los proyectos de Parques del Río y Cinturón Verde - Jardín Circunvalar, por cuanto a sus aportes en términos de movilidad y espacio público para Medellín y el Valle de Aburrá.

\section{Agradecimientos}

Extiéndase mi más caluroso agradecimiento, por toda su colaboración y apoyo a:

Sra. Sonia Astúa F. Coordinadora del Programa de Pasantías para la Movilidad Estudiantil Internacional del Instituto Tecnológico de Costa Rica y a todo el personal de Rectoría.

Sra. Claudia Madrizova M. Vicerrectora de la Vicerrectoría de Vida Estudiantil y Servicios Académicos del Instituto Tecnológico de Costa Rica.

Sr. Gustavo Rojas M. Director de la Escuela de Ingeniería en Construcción del Instituto Tecnológico de Costa Rica y a todo el personal docente y administrativo. 
Sr. Roberto Bayardelle M. Director de Planeación en la Empresa de Transporte Masivo del Valle de Aburrá Ltda.

Sr. Juan Diego Madrid A. Auxiliar Administrativo Administración Documental en la Empresa de Transporte Masivo del Valle de Aburrá Ltda., Biblioteca del Metro.

\section{Referencias}

[1] Colegio Federado de Ingenieros y de Arquitectos de Costa Rica (CFIA). (2013). Perfiles profesionales. Consultado en: http://www.cfia.or.cr/descargas_2013/formacion_profesional/perfil_civil_octubre13.pdf

[2] Instituto Nacional de Estadística y Censo. (2013). Estimaciones y Proyecciones de Población por sexo y edad 1950 - 2050. Primera Edición. Cuadro 2.1. Página 65. San José, Costa Rica. Consultado en: http://www.inec. go.cr/sites/default/files/documentos/poblacion/estimaciones_y_proyecciones_de_poblacion/publicaciones/ repoblacev1950-2050-01.pdf

[3] Cámara de Comercio de Medellín para Antioquia. (2011). Perfil socioeconómico de Medellín y el Valle de Aburrá. Tabla 1. Página 5. Medellín, Colombia. Consultado en: http://www.camaramedellin.com.co/site/ Portals/0/Documentos/2017/Publicaciones\%20regionales/17-3Perfil\%20ValledeAburra_Oct14.pdf

[4] Empresa de Transporte Masivo del Valle de Aburrá Ltda. (1980). Diseño preliminar (resumen). Medellín, Colombia.

[5] Empresa de Transporte Masivo del Valle de Aburrá Ltda. (2017). El Metro de Medellín aportó a la movilidad sostenible durante 2016. Noticias Metro. Medellín, Colombia. Consultado en: https://www.metrodemedellin. gov.co/al-día/noticias-metro/artmid/6905/articleid/131/el-metro-de-medell205n-aport211-a-la-movilidadsostenible-durante-2016

[6] Empresa de Transporte Masivo del Valle de Aburrá Ltda. (2017). La Cívica se extiende como el medio de pago unificado en el transporte de Medellín y el Área Metropolitana. Noticias Metro. Medellín, Colombia. Consultado en: https://www.metrodemedellin.gov.co/al-d\%C3\%ADa/noticias-metro/artmid/6905/articleid/391/la-c237vicase-extiende-como-medio-de-pago-unificado-en-el-transporte-de-medell237n-y-el-225rea-metropolitana

[7] Empresa de Transporte Masivo del Valle de Aburrá Ltda. (2015). Entre rieles y cables. Primera edición. Editorial Universo Centro. Bogotá, Colombia.

[8] Germán G. Madrid M. (Julio 2017). Entrevista personal. Medellín, Colombia.

[9] Empresa de Transporte Masivo del Valle de Aburrá Ltda. (2017). Historia. Medellín, Colombia. Consultado en: https://www.metrodemedellin.gov.co/qui\%C3\%A9nessomos/historia

[10] Empresa de Transporte Masivo del Valle de Aburrá Ltda. (2017). Se acerca el mantenimiento anual de los metrocables. Noticias Metro. Medellín, Colombia. Consultado en: https://www.metrodemedellin. gov.co/al-d\%C3\%ADa/noticias-metro/artmid/6905/articleid/212/se-acerca-el-mantenimiento-anual-de-losmetrocables

[11] Metroplús S.A. (2017). Historia. Medellín, Colombia. Consultado en: http://metroplus.gov.co/nuestra-empresa/ historia/

[12] Caracol Radio. (2014). Sistema Integrado de Transporte de Medellín exporta asesoría y conocimiento. Medellín, Colombia. Consultado en: http://caracol.com.co/radio/2014/02/16/regional/1392534720_083487.html

[13] Encicla. (2017). ¿Cómo funciona? Medellín, Colombia. Consultado en: http://www.encicla.gov.co/comofunciona/ 